\title{
¿Aceptación o control mental? Terapias de aceptación y mindfulness frente a las técnicas cognitivo-conductuales para la eliminación de pensamientos instrusos
}

\author{
Jorge Barraca Mairal \\ Universidad Camilo José Cela
}

\begin{abstract}
RESUMEN
Durante décadas los psicólogos clínicos han desarrollado procedimientos para extinguir, disminuir y/o cambiar los pensamientos intrusos (por ejemplo, la parada de pensamiento, la distracción o la reestructuración cognitiva). Sin embargo, hoy en día estas técnicas han sido cuestionadas tanto desde la investigación básica (experimentos sobre supresión de pensamiento) como desde el campo clínico aplicado. Como alternativa, las terapias de conducta de tercera generación proponen recurrir a los métodos de aceptación y mindfulness ante la aparición de este tipo de pensamientos. En el artículo se presenta un resumen sobre las técnicas cognitivo-conductuales tradicionales y se explica la incorporación de la aceptación y mindfulness en cuatro terapias de tercera generación. Las evidencias empíricas de estos dos enfoques de tratamiento se contraponen y se concluye que la aceptación y el mindfulness están consiguiendo unos resultados muy prometedores para el tratamiento de los pensamientos intrusos; no obstante, sigue siendo cierto que métodos tradicionales, como la reestructuración o la distracción focalizada, continúan mostrando su utilidad en muchos casos.
\end{abstract}

Palabras clave: pensamientos intrusos, cogniciones, supresión de pensamiento, terapias basadas en la aceptación y mindfulness, control mental, parada de pensamiento, reestructuración cognitiva.
AbStRaCt

For decades, clinicians have developed procedures in order to extinguish, dismiss or modify intrusive thoughts (e.g., thought stopping, distraction, or cognitive restructuring). However, nowadays these techniques have been questioned both in the basic research (thought suppression experiments) as well as in the clinical applied field. Alternatively, the thirdwave of behavioral therapy propose the employ of acceptance and mindfulness in the occurrence of such thoughts. This paper put forward an overview of the traditional cognitive-behavioral techniques and explains the integration of acceptance and mindfulness in four of the thirdgeneration therapies. Empirical evidence of these two treatment approaches are contrasted and we conclude that acceptance and mindfulness are obtaining very promising results for the treatment of intrusive thoughts; however, the fact remains that some traditional methods such as restructuring or focused distraction, continue to show their usefulness in many cases.

Keywords: : Intrusive thoughts, cognitions, thought suppression, acceptance and mindfulness-based therapies, mental control, thought stopping, cognitive restructuring. 
La facultad para controlar nuestra mente y dirigirla de forma voluntaria hacia aquellas tareas y contenidos que se desea en cada momento es, probablemente, un anhelo tan antiguo como el mismo ser humano. No obstante, gozar de esta destreza parece algo muy alejado de la cotidianidad. El terapeuta desearía recordar el nombre del paciente que tiene delante; el alumno, la respuesta a la pregunta del examen que poco antes había estudiado; la persona que necesita comprar una medicina, la calle donde está la farmacia de guardia y que retenía en la mente hasta hace sólo unos minutos. Por el contrario, la mujer de un marido infiel, desearía no recordar más ese engaño que ella le perdonó hace ya tiempo; el empleado, el comentario desagradable de su jefe; el conductor el accidente que le obligó a guardar reposo durante un mes. Desembarazarse de un recuerdo desagradable, que vuelve una y otra vez, no parece nada sencillo, como tampoco lo es concentrarse sin distraerse en una tarea o recordar algo importante justo cuando se desea. Esta asimetría en el control mental es un motivo común de malestar y la motivación que conduce a muchas personas a las consultas psicológicas.

Se ha calculado que, como promedio, en dieciséis horas de consciencia, tenemos unos 4.000 pensamientos, habitualmente con una duración de pocos segundos (Klinger, 1978, 1996). Un estudio reciente de Killingsworth y Gilbert (2010), con una amplia muestra de 2.250 participantes, ha sacado a la luz que la mayoría de las personas emplea un 46,9\% de las horas de vigilia a pensamientos que nada tienen que ver con lo que está haciendo en esos momentos. Dadas estas cuantías parece lógico que no se pueda evitar que estos pensamientos sean de todo tipo: agradables, neutros o desagradables, repentinos o progresivos, deseados o intrusos, realistas o absurdos, agresivos o bienintencionados, producto del azar o del esfuerzo de concentración.

Por otro lado, se ha defendido que, cuando los pensamientos no deseados alcanzan un nivel determinado, llegan a imposibilitar un funcionamiento normal o adaptado; este sería el caso en los estados depresivos, el trastorno por ansiedad generalizada, el obsesivo-compulsivo, el de estrés postraumático o el de algunas psicosis (Clark \& Purdon, 2009); no obstante, el papel etiológico de los pensamientos intrusos para estas y otras psicopatologías es un tema controvertido. También se ha planteado que no es tanto la frecuencia de estos pensamientos lo que tiene que ver con la aparición o mantenimiento de estos cuadros, sino su relación con emociones displacenteras (Wegner \& Zanakos, 1994), lo que llevaría a un esfuerzo crónico por librarse de ellos y a una mayor sensación de fracaso al no alcanzar este objetivo (Purdon, Rowa, \& Antony, 2005). Justamente, focalizarse sobre esos esfuerzos y verlos como el problema -en vez de en la aparición en sí de los pensamientos, su contenido, su frecuencia o intensidad - es una de las claves sobre las que pivota la intervención propuesta en una de las terapias de conducta de tercera generación: la Terapia de Aceptación y Compromiso (ACT) (Hayes, Strosahl, \& Wilson, 1999).

El deseo de desembarazarse de ciertos pensamientos es algo común no sólo en la población clínica, sino también entre la población 
normal (Shingler, 2009). En muchos casos, se intenta, sencillamente, «no pensar más en ello». Clark y Purdon (2009), por medio de una entrevista estructurada, encontraron que, entre los estudiantes universitarios, las estrategias más comunes ante los pensamientos intrusos fueron: tratar de distraerse, intentar racionalizarlo o comprobarlo, relajarse, parar el pensamiento $y$, también, no hacer nada. En este mismo estudio se observó que, al menos con población no clínica, los pensamientos de contenido obsesivo y de preocupación son comunes, y que las estrategias para librarse de ellos muchas veces no tienen los resultados deseados.

De hecho, la literatura científica ha encontrado reiteradamente que las estrategias encaminadas a librarse de los pensamientos incómodos llevan con frecuencia a resultados paradójicos, que consisten en el incremento en la tasa e intensidad de los pensamientos que tratan de evitarse (ver Rassin, 2005, para una completa revisión). Najmi \&Wegner (2008) han planteado que es posible que los intentos de supresión de los pensamientos sean un factor que complica y agrava muchos cuadros psicopatológicos, pues el esfuerzo por no estar en contacto determinados pensamientos supondría una carga cognitiva que minaría la capacidad para mantenerlos alejados y que conduciría, así, a un círculo vicioso de expectativas frustradas y mayor estrés ante su reaparición.

El objeto de este artículo es presentar el enfoque alternativo que las terapias de aceptación y mindfulness ofrecen para el manejo de los pensamientos y recuerdos indeseados. Para ello, se describen primero los resultados de la investigación sobre supresión de pensamien- to y se repasan los procedimientos cognitivoconductuales tradicionalmente empleados para el control de éstos, así como su efectividad. Por último, se explica cómo los abordan cuatro terapias de tercera generación y cuáles son sus resultados empíricos.

\section{El estudio experimental de la supresión de pensamiento}

Desde sus primeras publicaciones en la década de los ochenta, los estudios de laboratorio de Wegner y sus colaboradores han resultado una inspiración fundamental para multitud de clínicos que han cuestionado la utilidad de las estrategias directas de control mental. En un principio, Wegner, Schneider, Carter y White (1987) comprobaron la imposibilidad de los participantes de liberarse de una imagen mental (un oso blanco) durante cinco minutos cuando se les instruía específicamente en este sentido. Frente a lo que podría creerse antes de empezar el experimento, mantenerse libre del pensamiento resulta estadísticamente poco probable al cabo de sólo unos pocos minutos. Es más, tratar durante un tiempo de eliminarlo parecía provocar el que resurgiese con más frecuencia e intensidad en un segundo momento del experimento. En pocas palabras: la supresión voluntaria del pensamiento acababa tornándose contraproducente.

Wegner (1994) postuló la teoría del proceso irónico del control mental para explicar por qué los pensamientos que tratan de desecharse regresan una y otra vez a la conciencia. La teoría sostiene que en los esfuerzos de supresión intervienen dos procesos: uno consciente, operativo y costoso cognitivamente que se orienta 
a distraer la atención de los pensamientos no deseados, y otro inconsciente, de seguimiento y sin esfuerzo que, por un lado, vigila la aparición de esos mismos pensamientos, y, por otro, dispara la acción del proceso operativo en el caso de que resurjan los pensamientos evitados. Ambos procesos trabajan conjuntamente para asegurar el control, pero, irónicamente, al mantener la vigilancia constante, el segundo proceso provoca que el pensamiento indeseado permanezca siempre latente.

De acuerdo con este modelo, cuando se recurre a distintas estrategias distractoras (distracción no focalizada), como, por ejemplo, fijarse en lo que hay alrededor, tratar de pensar en otra cosa, procurar recordar una canción, etc. acaba por experimentarse un efecto rebote del pensamiento que trata de eliminarse, pues al final todos estos estímulos acaban convirtiéndose en claves de recuerdo. No obstante, si se recurre a una distracción focalizada, como, por ejemplo, sustituir continuamente el pensamiento del oso blanco por un pensamiento de un coche rojo, las posibilidades de sufrir el efecto rebote son menores, al menos a corto plazo (Salkovskis \& Campbell, 1994; Wegner et al., 1987).

Sea correcta o no la teoría irónica, el efecto rebote ha sido repetidamente estudiado experimentalmente (Abramowitz, Tolin, \& Street, 2001; Rassin, 2005; Wenzlaff \& Wegner, 2000) y, en general, aunque no sin alguna excepción, replicado. Por otro lado, se ha comprobado que este efecto resulta más frecuente cuanto mayor es la carga mental. Además, sus efectos no son pasajeros: siguen interfiriendo con la actividad tiempo después de efectuado el experimento (Wenger \& Erber, 1992).
La investigación ha demostrado también que, junto con la carga mental, el estado de ánimo influye en la capacidad de supresión: los sujetos depresivos muestran menos capacidad para librarse de pensamientos intrusos. Igualmente, se produce una interacción con el tipo de pensamientos: si son congruentes con el estado anímico resultan más difíciles de desechar. Por eso, a los sujetos depresivos les cuesta significativamente más librarse de pensamientos tristes que de pensamientos alegres (Howell \& Conway, 1992; Purdon \& Clark, 2000).

Algunos otros autores (cf. Anderson \& Green, 2001; Hertel \& Calcaterra, 2005) han propuesto estrategias distintas — como el paradigma «pensar/no pensar»—, en la que se instruyen procedimientos de supresión con otro tipo de atención focalizada. En principio, estos métodos parecen haber resultado más eficaces para lograr una supresión del pensamiento. Sin embargo, es cierto que, salvo en algunas excepciones (por ejemplo, Luciano \& Algarabel, 2007), se han empleado en estos trabajos palabras neutras y sin significado emocional para los participantes.

\section{La intervención cognitivo-conductual para} los pensamientos intrusos e inadaptados

Tradicionalmente, los terapeutas cognitivoconductuales han sido instruidos en la necesidad de ayudar a sus pacientes a librarse de sus pensamientos intrusos o inadaptados $y$, para tal fin, se han desarrollado distintas técnicas. Estos procedimientos se idearon bastante antes de que Wegner y sus colaboradores empezaran los experimentos sobre supresión de pensamiento; sin embargo, la revisión que 
ahora se hace de estos métodos incluye algunas de estas aportaciones posteriores.

Parada de pensamiento. El primero procedimiento sistemático planteado desde la modificación de conducta para el control de los pensamientos desagradables y los recuerdos ingratos fue publicado por Wolpe (1958). Su método de parada de pensamiento es un entrenamiento medianamente complejo, bien estructurado en fases y que requiere una notable implicación y motivación por parte del paciente. Se entrena primero en sesión, pero son imprescindibles las prácticas posteriores en el entorno natural del sujeto.

Básicamente, el método consiste en la detección y retirada inmediata de pensamientos inadecuados, desadaptados y desagradables que previamente se han concretado en la terapia. En un primer momento, la retirada se lleva a cabo por medio de un estímulo físico verbal (por ejemplo, gritar «stop») y/o corporal (palmada simultánea, golpe en la muñeca con una goma elástica) de intensidad tal que corte al instante el pensamiento. Más adelante, será suficiente con que este estímulo se reproduzca en imaginación. La técnica se complementa con la prescripción de una actividad altamente distractora inmediatamente posterior a la parada del pensamiento (por ejemplo, hablar con otra persona, hacer cuentas mentales, repetirse un listado de actividades o frases positivas, ver un vídeo, etc.) y que ha sido seleccionada por su utilidad en el caso concreto. La incorporación de este último componente de sustitución de los pensamientos intrusos y su entrenamiento encubierto ha llevado a que algunos autores denominen a esta técnica aserción encubierta (McKay, Davis, \& Fanning, 1985).
Existen varias teorías para justificar el procedimiento de parada de pensamiento (puede consultarse Bakker, 2008, para una revisión). Por un lado, la reiteración sistemática de este proceso facilitaría un condicionamiento clásico de modo que, en cuanto surge el pensamiento inadecuado, tras varios ensayos, se interrumpe enseguida por la contingencia establecida (aparición - parada inmediata). Otra posibilidad es que la repetición de la técnica favorezca una habituación a los pensamientos previamente desagradables, ya que al tener que ensayarse muchas veces la exposición a los mismos se hace inevitable. Más recientemente, también se han propuesto algunas teorías cognitivas.

Desde su aparición y divulgación en textos (Rimm \& Masters, 1979; Wolpe, 1973) la parada de pensamiento ha sido muy bien aceptada por los psicólogos en práctica clínica y se ha utilizado innumerables veces. Existen muchos estudios que evidencian su eficacia en distintos cuadros psicopatológicos (Wolpe, 1992), pero también es cierto que su uso ha sido muy cuestionado, especialmente para la intervención en los cuadros obsesivos (Hannan \& Tolin, 2005). Una revisión sistemática de su utilidad así como de importantes recomendaciones sobre su uso en la actualidad pueden encontrarse en Bakker (2009).

Reestructuración cognitiva. Frente a la estrategia de desechar de la conciencia determinados pensamientos directa y rápidamente, desde la terapia cognitiva se planteó la posibilidad de rebatirlos para conseguir que disminuyese su intensidad $o$, al menos, su credibilidad y, como consecuencia, sus efectos sobre el estado de ánimo. Distintos tipos de reestructu- 
raciones cognitivas como métodos para contrarrestar los pensamientos intrusos son sugeridos en los trabajos de Albert Ellis (Ellis, 1962; Ellis \& Bernard, 1985; Ellis \& Dryden, 1997) y de Aaron T. Beck (Beck, 1976; Beck, Emery, \& Greenberg, 1985). Más específicamente, Beck, Rush, Shaw y Emery (1979/1983), al explicar el objetivo de las técnicas cognitivas para el tratamiento de la depresión, mencionan que el método que proponen tiene como primer objetivo: «controlar los pensamientos (cogniciones) automáticos negativos» (p. 13).

Aunque la reestructuración cognitiva es muy variada en su puesta en práctica, de acuerdo con las intervenciones más difundidas podría decirse que, una vez identificados los elementos cognitivos, "el objetivo es poder ayudar al paciente a modificarlos con el fin de poder hacer más adaptativo y agradable su funcionamiento en el medio» (Buela-Casal, Sierra, \& Vera-Villarroel, 2001, p. 207). Las estrategias para lograrlo son distintas de acuerdo con el tipo de intervención cognitiva; no obstante, habitualmente en estos entrenamientos se siguen estas fases: (1) ayudar al paciente a establecer relaciones entre su cognición, sus emociones y sus conductas; (2) ayudar al paciente a identificar los pensamientos más disfuncionales o irracionales, o sus procesos cognitivos más desadaptativos (por ejemplo, captar sus debo-perturbadores, sus pensamientos dicotómicos, sus inferencias arbitrarias, sus abstracciones selectivas, sus maximizaciones, etc.); y (3) modificar estas cogniciones por medio de distintos métodos, bien de corte más cognitivo (por ejemplo, comprobaciones lógicas y empíricas, reatribuciones de causas y responsabilidades, conceptualizaciones alter- nativas, llevar al absurdo las ideas sostenidas, etc.), bien por medio de procedimientos conductuales (como la exposición, las tareas para casa, el role-playing, los refuerzos y castigos, etc.) (Martin \& Pear, 2008).

Existen evidencias sólidas de la utilidad de estas técnicas para conseguir mejorías en los pacientes, aunque los resultados están asociados a la recuperación, en general, de los cuadros depresivos (Dobson, 1989) más que a la desaparición completa o al cambio definitivo en los pensamientos intrusos. Por otro lado, sigue en cuestión cuál es el valor causal de los componentes cognitivos, frente a los conductuales, en la eficacia de terapias cognitivas como la de Beck (Gortner, Gollan, Dobson, \& Jacobson, 1998; Jacobson et al., 1996).

Es importante señalar que la investigación sobre los cambios producidos en las ideas irracionales o en los patrones de pensamiento no ha dejado claro que estos cambios - que, además, habitualmente son inestables - se deban justamente a las técnicas cognitivas mismas. Tal y como denuncian Kanter et al. (2010) - y también Hayes, Luoma, Bond, Masuda y Lillis (2006) - , el estatus empírico de las técnicas de reestructuración cognitiva y la posibilidad de que aporten un diferencial de eficacia a las técnicas meramente conductuales continúa siendo un tema muy controvertido, a pesar de los años de investigación en el campo.

Otras técnicas. Desde la terapia cognitivoconductual se han propuesto otras modalidades de intervención para el tratamiento de los pensamientos intrusos. Sin pretender ser exhaustivos podría mencionarse entrenamientos como la relajación y la respiración, la distrac- 
ción sin parada de pensamiento y la intención paradójica.

La relajación muscular progresiva o la respiración diafragmática se han propuesto en ocasiones; no obstante, casi siempre en combinación con otras técnicas, por lo que resulta difícil valorar su eficacia. Como intervención única, Salkovskis \& Reynolds (1994) hallaron que la distracción por medio de técnicas de respiración y ejercicios de relajación resultaba eficaz para reducir pensamientos intrusos sobre las ganas de fumar.

La distracción sin parada de pensamiento ha sido empleada como método para enfrentarse a pensamientos intrusos egodistónico de contenido sexual o agresivo (McKay et al., 1985). En esta técnica cuando aparece el pensamiento desagradable, el sujeto inicia otra actividad distractora, como leer una lista de pensamientos agradables alternativos previamente pensada y redactada. Por tanto, se busca controlar el malestar por medio del remplazo de unos pensamientos por otros, siempre dentro de una situación determinada. A diferencia de lo que sucede en los métodos experimentales de supresión del pensamiento (como la distracción focalizada, a la que podría asociarse), en este caso hay una estrategia ya determinada (pensamientos alternativos redactados) y ensayada.

Porúltimo, con el procedimiento de intención paradójica (Frankl, 1967) la estrategia es justamente la contraria a las planteadas hasta ahora: se trata de prescribir el síntoma mismo a fin de aumentar, al cabo, el control sobre él o conseguir así indirectamente que desaparezca. Para el caso de los pensamientos intrusos, en esta técnica el terapeuta prescribe al paciente que mantenga en la mente, sin distraerse, aquellos contenidos mentales que hasta ese momento ha tratado de evitar. Sin embargo, el paciente, al no ser capaz de aguantar sin distraerse en algún momento o al habituarse por la saturación de esos pensamientos, acabaría por mejorar. La intención paradójica se ha incorporado como una técnica más en distintas intervenciones cognitivo-conductuales, si bien hay que advertir que hay grupos de pacientes con los cuales deben tenerse especiales precauciones y su utilidad resulta controvertida (Down \& Swoboda, 1984). Además, una revisión de trabajos en las que se empleó para pensamientos obsesivos e intrusos mostró resultados muy modestos, excepto cuando se acompañaba de procedimientos de exposición (Foa \& Franklin, 2002).

En suma, existen aún pocas evidencias sobre los resultados experimentales de la relajación, la distracción y la intención paradójica para el caso concreto de la desaparición de los pensamientos intrusos; además, casi siempre estos métodos han añadido otros componentes (por ejemplo, exposición, técnicas cognitivas) que no permiten ofrecer conclusiones sobre su eficacia diferencial.

\section{Las terapias de aceptación y mindfulness} y su intervención en el control de los pensa-

\section{mientos intrusos}

Los trabajos de Wegner y sus colaboradores antes referidos - como también los de Clark, Ball y Pape (1991) y Cioffi y Holloway (1993)han sido recogidos por Hayes et al. (1999, pp. 60-61) para aportar argumentos a favor de la intervención que plantea la ACT. No obstante, no sólo se estiman útiles para esta intervención, sino también en otras de las denominadas terapias 
de tercera generación o de tercera ola en la terapia de conducta (Hayes, 2004), que, además de ACT, incluiría, fundamentalmente, la Psicoterapia Analítico Funcional (FAP) (Kohlenberg \& Tsai, 1991), la Terapia de Activación Conductual (BA) (Jacobson, Martell, \& Dimidjian, 2001), la Terapia Integral de Pareja (IBCT) (Jacobson \& Christensen, 1996), la Terapia Dialéctica Comportamental (DBT) (Linehan, 1993) y la Terapia Cognitiva con base en la Atención Plena o Mindfulness (MBCT) (Segal, Williams \& Teasdale, 2002).

De acuerdo con la delimitación de Hayes (2004), estos desarrollos terapéuticos tienen en común un planteamiento contextual, otorgan un énfasis singular al análisis de la funcionalidad de la conducta, toman la flexibilidad como criterio de adaptación ante los problemas (en vez de promover una intervención directa sobre los eventos) y despsiquiatrizan o desmedicalizan las categorías diagnósticas tradicionales. No obstante, aunque todas ellas poseen evidentes puntos de unión (Barraca, 2006; Pérez Álvarez, 2006), también es innegable que presentan claras diferencias en sus formatos, tiempos de intervención, principios rectores, habilidades clínicas, etc. (Barraca, 2009).

En el tema en concreto del manejo de los pensamientos intrusos las terapias de tercera generación poseen igualmente algunos actitudes y planteamientos comunes (en particular, el empleo del mindfulness y de procedimientos basados en la aceptación), pero también distinciones. A continuación, se mencionarán cuáles son las estrategias de cuatro de estas terapias ( $A C T, D B T, M B C T$ y $B A$ ) ante la aparición de pensamientos juzgados como inapropiados o dolorosos.
ACT. De entrada, como postulado, la ACT plantea que el manejo de la experiencia interna se debe llevar a cabo de forma distinta a como se hace en la conducta externa. Se habla, por ello, de la regla de los eventos privados (Hayes et al., 1999, p. 120), que sería opuesta a la de los eventos externos. Mientras que para el control de estos últimos la conducta intencional sería apropiada, no pasaría otro tanto para aquéllos. «En el mundo que hay dentro (de la piel), la regla viene a decir: si no estás dispuesto a tenerlo, entonces lo tienes» (p. 121).

Coherentemente con este postulado, la experiencia interna -cualquiera que esta sea: pensamientos, recuerdos, sensaciones, etc.- debe dejarse tal cual es, sin intentar detenerla, controlarla, modificarla, magnificarla, minimizarla, etc. (Hayes et al., 1999). Es más, el intento repetido por alterar esta experiencia, de procurar cambiarla y no lograrlo llevaría a un problema psicopatológico denominado trastorno de evitación experiencial (Hayes, Wilson, Gifford, Follette, \& Strosahl, 1996). En suma, de acuerdo con ACT los intentos reiterados por lograr controlar la mente conducirían al cabo a un mayor malestar.

Desde este modelo, la alternativa ante los pensamientos intrusos (o ante sentimientos, sensaciones, recuerdos, etc. desagradables) es la aceptación radical o incondicional de los mismos, sean como sean de frecuentes o intensos y sea cual sea su contenido. Esto implica que, por ejemplo, el paciente que sufre trastorno de angustia debe procurar aceptar sus crisis de ansiedad, y sentirlas como son; el depresivo su tristeza; el obsesivo sus pensamientos obsesivos; el psicótico sus voces y sus alucinaciones; o el paciente de dolores crónicos su dolor, 
cesando así su lucha permanente por contralo. Sin embargo, no se trata de una aceptación pasiva (de una resignación ante el síntoma), sino del abandono de aquellos intentos de cambiar los pensamientos que llevan a bloquear la vida. El terapeuta de la ACT animará y conducirá al cliente a centrar sus esfuerzos en recuperar una vida con sentido, una encaminada a contactar de nuevo con las cosas valiosas para la persona, y que se concreta en unos objetivos específicos de acción dentro del terreno de la conducta manifiesta.

Como ayuda en este proceso, la ACT emplea diversos procesos (Hayes et al., 1999; Hayes et al., 2006), como la aceptación, la defusión cognitiva, el estar presente, el concebir el self como contexto, el trabajo con los valores y la acción comprometida. A su vez estos procesos se facilitan por medio de ejercicios vivenciales, metáforas y paradojas, y no tanto por explicaciones lógicas o discursivas.

La defusión cognitiva es un componente de la ACT especialmente relacionado con el manejo de los pensamientos intrusos. Una de sus metas consiste en lograr la deliteralización de las palabras-conceptos, es decir, hacer ver que los pensamientos son sólo pensamientos o sólo recuerdos, y que no deben confundirse con sus referentes, se entiende: sucesos reales presentes que vuelven imposible actuar de acuerdo con lo valorado. Este proceso de deliteralización se puede lograr por varios métodos como la contemplación desapasionada del pensamiento, el repetirlo en alto hasta que sólo quede el sonido (sin sentido), el contemplarlo como un evento externo del que se puede jugar su forma, color, velocidad o aspecto, el agradecer a la mente haber proporcionado un pensamiento tan curioso e interesante, etc. (Hayes et al., 2006). Tales actitudes conducirán a una actitud más objetiva y distanciada respecto a los pensamientos (pues es más cierto darse cuenta de que se está pensando: «ahora estoy pensando que soy un idiota», en vez de creer taxativamente: «soy un idiota»). Por ello, la defusión conduce a disminuir la credibilidad o el apego a los pensamientos, aunque no necesariamente altera su frecuencia.

Simultáneamente, otros procesos para ayudar en este camino son el estar presente y el self como contexto, que puede integrarse en la actitud de mindfulness. Como éste es la base de la siguiente terapia, se explica en el próximo modelo de intervención.

MBCT. La divulgación del mindfulness (o atención consciente o plena) es producto, fundamentalmente, de la labor divulgadora de Kabat-Zinn (1990), y dentro de la tradición cognitivo-conductual del texto de Segal, Teasdale, \&Williams (2002), aunque también ha sido propuesto dentro de la DBT por Linehan (1993) y en la ACT (Hayes, Jacobson, Follette, \& Dougher, 1994). El mindfulness es un procedimiento que hunde sus raíces en la meditación de tradición budista Zen. Por tanto, desde una perspectiva filosófica-religiosa, no sería tanto una técnica que se lleva a cabo en un momento particular, sino una actitud que abarcaría todo el tiempo y que se correspondería con una forma de estar $y$ de contemplar el mundo. No obstante, aquí se entiende como un procedimiento concreto, un instrumento para el manejo de los pensamientos intrusos. En este sentido, su inclusión en la terapia psicológica es reciente. 
El mindfulness como técnica poseería una serie de características que, siguiendo a Vallejo (2006), incluirían: (a) centrarse en el momento presente; (b) apertura a la experiencia y a los hechos (se entiende sin interpretaciones o mediaciones del lenguaje); (c) aceptación radical y no valorativa de la experiencia; (d) elección de las experiencias en que centrarse, mirar, actuar o implicarse; y (e) renuncia al control directo de las emociones, reacciones, sentimientos o pensamientos, para permitirles ser tal cual son, sin pretender cambiarlos.

Teasdale, Segal, Williams, Ridgeway, Soulsby y Lau (2000) presentan evidencias de que la realización de un taller de mindfulness de ocho semanas (de dos a dos horas y media, y con una práctica en casa de cuarenta y cinco minutos al día) era una estrategia eficaz para evitar las recaídas en personas que habían sufrido dos más episodios de depresión grave y de las que ya se habían recuperado. Desde su punto de vista, la práctica del mindfulness al que comprometía el taller ayudaba a vérselas más adecuadamente con los pensamientos que preceden de forma característica a las recaídas depresivas. Este tipo de pensamientos intrusos sería mejor manejado gracias al mindfulness porque esta técnica favorecería un distanciamiento del contenido del pensamiento $y$, por tanto, una menor credibilidad del mismo, todo lo cual conduciría a verse menos afectado por su presencia y, al cabo, a evitar la recaída.

Es importante señalar que esta práctica de mindfulness no predice que los pensamientos intrusos y rumiantes de la depresión serán menos, sino que la vivencia y el apego a éstos serán distintos.
BA. De los protocolos existentes hoy en día para la aplicación de las técnicas de activación conductual —el de Lejuez y sus colaboradores (Lejuez, Hopko, \& Hopko, 2001; Lejuez, Hopko, Acierno, Daughters, \& Pagoto, 2011) y el de Martel y sus colaboradores (Martell, et al., 2001; Martell, Dimidjian, \& Herman-Dunn, 2010)—, sólo en este último se proponen estrategias para afrontar los pensamientos intrusos negativos y, más en concreto, la rumiación depresiva. No es que Lejuez y sus colegas no den importancia a esta actividad cognitiva, tan relevante en el caso de los estados depresivos, pues de hecho, valora como clave diagnóstica, pero lo desestiman una intervención directa sobre ella al opinarse que desaparecerá por sí sola cuando mejore el estado ánimo del cliente. En el caso del protocolo de Martell y sus colaboradores no se niega que eso mismo pueda suceder, pero se cree que, en ocasiones, las estrategias de activación (la monitorización de la actividad, la evaluación de metas y valores vitales, la programación jerárquica de actividades reforzantes, el entrenamiento en habilidades sociales o el control de contingencias) pueden verse afectados negativamente por la actividad cognitiva, por lo que las posibilidades de mejorar anímicamente serán menores si no se trata.

Sin embargo, lo más destacado es que esta actividad no se aborda de forma distinta a la de cualquier otra conducta manifiesta y, por tanto, se valora con el mismo análisis funcional que el resto. Esto es: trata de determinarse al servicio de qué están los pensamientos, y no tanto su lógica o contenido. Así, se lleva a cabo un análisis contextual de la rumia depresiva en vez de acometer ningún tipo de reestructuración 
cognitiva (Kanter et al., 2010). El terapeuta de BA no discutirá la veracidad de los pensamientos del pacientes, no tratará de rebatirlos, sino que considerará si son formas de evitación de ciertas situaciones, cómo se siente el paciente tras un periodo de rumia, qué otras cosas (en vez de rumiar) podría hacer, etc. (Martell et al., 2001). Desde un enfoque genuinamente contextual, los pensamientos no puede verse sino como una consecuencia de las contingencias a las que está el sujeto.

Junto con este enfoque, los autores de BA proponen también el empleo del mindfulness, aunque no tanto con los ejercicios formales de un entrenamiento convencional, sino, sobre todo, con la idea de que cuando se realice una actividad la atención se dirija a ella y no hacia los contenidos mentales que, simultáneamente, pueden aparecer. En suma, ante la aparición de pensamientos negativos intrusos se invita "atender a la experiencia" presente (Martell et al., 2001, p. 124) y percatarse de lo que se está haciendo y de lo que se tiene alrededor, focalizándose en lo que llega desde los sentidos.

DBT. Dentro de la amplia terapia desarrollada por Linehan (1993) para el tratamiento del Trastorno Límite de la Personalidad, el uso de algunas estrategias para manejar pensamientos intrusos se imponía por razón de la frecuencia de estos problemas en este tipo de pacientes (en particular, la ideación suicida). Como ya se ha adelantado, en DBT se recomienda el empleo del mindfulness para experimentar las vivencias tal y como son, y no distorsionadas desde la interpretación catastrofista o negativa propia de las personas que sufren este trastorno de la personalidad.
La práctica del mindfulness no se restringe aquí al manejo de los pensamientos distorsionados e intrusos, sino que se concibe como un entrenamiento útil para ganar habilidades tendentes a disminuir las conductas suicidas o parasuicidas, así como para eliminar otras acciones que interfieren con una vida sana y productiva (por ejemplo, abuso de sustancias, trastornos de la alimentación, cambios constantes de vivienda o de trabajo, etc.). Junto con el mindfulness, Linehan (1993) también propone entrenamientos concretos en distintos ámbitos, pues la DBT es una terapia con componentes de la terapia conductual clásica. Esta integración a veces ha llevado a algunas contradicciones, ya que mientras en unos casos (con el mindfulness) se propone la aceptación de los pensamientos tal cual son, en otros tratan de rebatirse con entrenamientos activos para regular las emociones, técnicas para incrementar la tolerancia al estrés y técnicas encaminadas a ganar eficacia interpersonal (Holmes, Georgescu, \& Liles, 2006).

Además del mindfulness, Linehan (1993) aconseja que los terapeutas validen permanentemente a sus pacientes, de forma que estos también acaben haciéndolo consigo mismos. Los procedimientos de validación tienen una conexión directa con los de aceptación, si bien se vinculan a la aceptación personal; es decir, a la legitimación de la forma de ser, los pensamientos, sentimientos o reacciones que uno tiene, más que a la aceptación en general de lo que la vida (o la mente) quiera traer. También en esta línea, en los grupos terapéuticos de habilidades que se llevan a cabo en DBT, se enfatiza que los pensamientos (y sentimientos) son respuestas 
privadas que acompañan de forma natural a la persona, y no algo que obligue a actuar de una manera determinada (Holmes et al., 2006).

\section{Evidencias empíricas}

La mayoría de las investigaciones efectuadas sobre estas terapias han analizado su eficacia como tratamientos de distintos trastornos psicopatológicos más que su utilidad para eliminar un posible síntoma, como es la intrusión de pensamientos indeseados. $Y$ el hecho de que, a día de hoy, se haya comprobado que la DBT es un tratamiento probablemente eficaz para el trastorno límite de la personalidad (Quiroga \& Errasti, 2003) o que la BA sea un tratamiento bien establecido para el trastorno depresivo mayor (Mazzucchelli, Kane, \& Rees, 2009) no necesariamente implica que todos los síntomas de estos cuadros desaparezcan tras la intervención, o que resulten efectivas las técnicas usadas específicamente para el problema de los pensamientos intrusos o la rumia depresiva.

Por otro lado, meta-análisis como los de Öst (2008) y Powers, Zum Vörde Sive Vörding y Emmelkamp (2009) revelan que es aún pronto para afirmar que las terapias de tercera generación en conjunto sean, a día de hoy, una alternativa a los tradicionales métodos cognitivo-conductuales. Es verdad que estos meta-análisis han sido cuestionados respectivamente por Gaudiano (2009) y por Levin y Hayes (2009), y que la ACT y otras terapias de la tercera generación están reuniendo día a día un mayor respaldo empírico en estudios aleatorizados controlados (cfr. Hayes et al. 2004).
Existen algunos trabajos experimentales más específicos que sí han comparado la eficacia para el control de los pensamientos de las técnicas basadas en la aceptación o en el mindfulness (procedimientos, como se ha visto, comunes en $A C T, B A, D B T$ y $M B C T$ ) frente a los métodos convencionalmente usados en la investigación (supresión de pensamiento, distracción -focalizada o indiferenciada- y reevaluación). Es una síntesis únicamente de estas investigaciones la que se incluye en este apartado.

Marcks y Woods (2005) a través de dos estudios empíricos demostraron que los métodos basados en la aceptación son más recomendables que los de supresión para pensamientos intrusos relevantes de la propia vida. Según su investigación, los esfuerzos de evitación de los pensamientos se asociaron a distintas condiciones psicopatológicas, provocaron mayor malestar subjetivo e, incluso, un efecto rebote de la frecuencia e intensidad de los pensamientos. Los autores concluyen que aquellas personas que, de forma más habitual, tratan directamente de suprimir los pensamientos intrusos relevantes cada vez se estresan más y sufren mayor necesidad de hacer algo respecto a estos pensamientos. En cambio, aquellos que de forma natural los aceptan presentan menos sintomatología depresiva, menos ansiedad y menos obsesiones. Es importante señalar que, en su segundo estudio, encontraron que las personas que se sirvieron de la aceptación bajaron su malestar, pero no la tasa de pensamientos intrusos, lo que resulta coherente con el modelo de ACT. En un trabajo publicado posteriormente (Marcks \& Woods, 2007), de nuevo se corroboró que, al contar e imaginar un accidente de tráfico de una persona querida, 
los que optaron por la estrategia de la aceptación sufrieron menos intrusiones, menos ansiedad y menos evaluaciones negativas que los que trataron de suprimirla.

En pacientes con diagnóstico obsesivocompulsivo, Najmi, Riemann, \& Wegner (2009) comprobaron que los sujetos que trataron de suprimir pensamientos clínicamente significativos vivieron mayor malestar que aquellos del grupo de aceptación; no obstante, estos últimos no se diferenciaron de los participantes del grupo instruido con procedimientos de distracción focalizada. Salkovsky, Thorpe, Wahle, Wroe y Forrester (2003) ya habían mostrado la eficacia en pacientes obsesivos de la distracción focalizada, frente a métodos como la neutralización de los pensamientos.

Resultados similares de eficacia entre métodos de aceptación y de atención focalizada ha sido igualmente encontrado en otras investigaciones (Luciano \& Algarabel, 2007), si bien con matices, pues este efecto se ve determinado por el contenido de los pensamientos alternativos en los que hay que focalizarse (Kimura, 2004).

Los estudios que han comparado estrategias como la supresión, la reevaluación de la situación y la aceptación han encontrado que la reeavaluación y la aceptación son claramente superiores a la supresión, aunque entre ellos es difícil establecer diferencias en la eficacia. La estrategia de reevaluación —al menos por su descripción en algunos trabajos- puede asimilarse a una forma breve de reestructuración cognitiva. Así, por ejemplo, en el estudio de Hoffman, Heering, Sawyer, \& Asnaani (2009), en que se pedía a los participantes que se controlasen pues, en breve, tendrían que improvi- sar ante una cámara un discurso sobre temas controvertidos, a los sujetos del grupo de reevaluación se les invitaba a tener una actitud realista y considerar que la situación a la que se iban a enfrentar realmente no representaba una amenaza, que, aunque pudiese crear cierto malestar, al fin no se trataba sino de un experimento y que su participación no tendría consecuencias negativas de ningún tipo.

En línea igualmente con las estrategias de reevaluación podría citarse la conclusión expuesta por Magee y Teachman (2007) que, por medio de una metodología de entrevista, demostraron la importancia de la atribución que el sujeto hacia ante su fracaso a la hora de suprimir un pensamiento: mientras que en unos casos las atribuciones era negativas y autoculpabilizadoras (por ejemplo, «no logro quitármelo de la cabeza porque hay algo malo en mi mente»; «porque estos pensamientos tienen demasiada fuerza») en otros casos se elaboraban visiones mucho más positivas (por ejemplo «no logro quitármelo justamente porque el examinador me dijo que no pensara en ello»; "porque la mente funciona así»). Este último tipo de atribuciones reducía significativamente la reaparición de los contenidos mentales evitados $y$, además, el estrés de la situación.

Ya se mencionó que en ACT la aceptación y la defusión cognitiva se facilita a través de ejercicios, como los encaminados a lograr la deliteralización. Uno de ellos consiste en repetir y repetir rápidamente una palabra (o un pensamiento) hasta que este deja de tener ningún sentido. En varios experimentos diseñados por Masuda y sus colaboradores (Masuda, Hayes, Sackett, \& Twohig, 2004; Masuda, Hayes, Two- 
hig, Drossel, Lillis, \& Washio, 2009; Masuda, Twohig, Storno, Feinstein, Chou, \& Wendell, 2010) se comprobó que la aplicación de estos procedimientos reducía la credibilidad y el malestar de los pensamientos negativos sobre uno mismo y favorecían su manejo. Frente a otras técnicas como tareas de distracción (lectura de un tema neutro), la respiración abdominal y la atención a pensamientos agradables, la deliteralización con el método propuesto por Hayes et al. (1999) resultó lo más eficaz para restar malestar y credibilidad al pensamiento.

Existe también evidencia específica respecto al empleo de técnicas de mindfulness para contrarrestar pensamientos obsesivos e intrusos. En una investigación con un diseño de serie de casos, Wilkinson-Tough, Bocci, Thome y Herlihy (2010) encontraron que todos sus participantes aquejados de pensamientos obsesivos intrusos redujeron sus puntuaciones en la escala de Yale-Brown a niveles subclínicos tras seis sesiones de un entrenamiento en mindfulness (acompañada de práctica diaria), ganancias que, en conjunto, se mantuvieron en el seguimiento. Un análisis cualitativo tras el procedimiento sugiere que habilidades inherentes al minfulness, como la observación, el ser consciente o la aceptación resultaron útiles de cara al manejo de la fusión pensamientoacción o la supresión de pensamientos.

Anteriormente, Hanstead, Gidron, \& Nyklíek (2008) con una muestra de 17 pacientes obsesivo-compulsivos asignados a una lista de espera control o a un tratamiento con ocho sesiones de mindfulness también encontraron que los pensamientos obsesivos se redujeron significativamente tras el entrenamiento $y$ que, como en el caso de Wilkinson-Tough et al. (2010), la fusión pensamiento-acción se redujo considerablemente, al igual que aumentó la capacidad de dejar ir (o desatender), coveniente para el manejo de esta problemática.

Hepburn, Crane, Barnhofer, Duggan, Fennell, \& Williams (2009) comprobaron que procedimientos de mindfulness resultaron eficaces para disminuir la supresión de pensamientos en pacientes suicidas, lo que está asociado a una menor vulnerabilidad. $Y$ en pacientes con trastorno bipolar, Miklowitz et al. (2009) y Williams et al. (2008) han encontrado que la MBCT facilitó la disminución de síntomas depresivos y ansiosos, entre los que se encontraban la ideación suicida.

Feldman, Greeson, \& Senville (2010), con una muestra de 190 mujeres, compararon un método de mindfulness (centrado en la respiración) con la relajación muscular y una meditación (centrada en la bondad) para evaluar el descentramiento, la frecuencia de pensamientos repetitivos y el grado de reacción negativa a los pensamientos. Las participantes en la condición de mindfulness fueron capaces de descentrarse en mayor grado; además, su asociación entre pensamientos repetitivos y reacciones negativas a los pensamientos resultó más débil. En suma, los resultados de este estudio experimental indican que el mindfulness de respiración puede ayudar a reducir la reactividad a pensamientos repetitivos.

Respecto al pensamiento rumiante crónico, hay al menos un trabajo empírico (Harrington, 2008) que ha revelado que un entrenamiento basado en la aceptación suponía una alternativa prometedora frente a una técnica conven- 
cional de control del pensamiento. No obstante, la autora reconoce que los resultados de este trabajo deben tomarse con cautela por los pequeños efectos que tuvo la intervención y lo limitado de la muestra.

Por último, no debe dejar de mencionarse la investigación de Dunn, Billotti, Murphy y Dalgleish (2009) en la que se usó un video de accidentes de tráfico y se obtuvieron medidas de los participantes de indicadores fisiológicos (tasa cardiaca, respuesta electrodermal) y cognitivos (recuerdo, probabilidad de intrusiones), tanto inmediatamente como al cabo de una semana. Contrariamente a lo hallado en la mayoría de los trabajos, los participantes en la condición de aceptación presentaron efectos más negativos (menos control fisiológico, más intensidad emocional y más recuerdo) que aquellos que se sirvieron de métodos directos de supresión.

\section{Conclusiones}

En conjunto, y con las excepciones señaladas, la revisión empírica arroja unos resultados muy prometedores para las técnicas de aceptación y mindfulness de cara al manejo de los pensamientos y la respuesta emocional asociada a éstos. Sin embargo, no es menos cierto que otras estrategias - como la distracción focalizada o la reevaluación - orientadas a desechar y cambiar los pensamientos intrusos y dolorosos también muestran buenos resultados. Por tanto, con el estado actual de la cuestión, es prematuro asegurar que los primeros deben desplazar a los métodos más convencionales. Creemos que no es muy desencaminado afirmar que, al menos en el terreno experimental, la partida está ahora mismo en tablas.
No obstante, si se atiende al interés que los métodos basados en la aceptación o el mindfulness está despertando en los clínicos de todo el mundo, es fácil que la balanza empiece a inclinarse a favor de la incorporación del enfoque de la tercera generación. Aparentemente, muchos terapeutas parecen encontrar en estas nuevas técnicas los métodos que echaban en falta para ayudar a sus pacientes para vérselas con determinados pensamientos. Por supuesto, este no es un argumento científico, y lo importante es que esa hipotética utilidad clínica se corrobore a través de investigaciones más amplias y mejor controladas. En particular, uno de los puntos a mejorar en la investigación consiste en la inclusión de seguimientos a largo plazo de la eficacia de las distintas técnicas. Hasta ahora los estudios analizan mayoritariamente qué pasa unos minutos después de emplear una estrategia u otra, o cuál es su resultado tras una o pocas semanas. Es evidente que con datos a tan corto plazo no puede concluirse qué estrategias son mejores desde el punto de vista clínico, en el que lo importante son los resultados a meses y años vista.

Por otra parte, es un error no establecer diferencias entre los métodos empleados en la literatura científica de supresión de pensamiento y los propuestos en la clínica conductual. De hecho, Bakker (2009) ha señalado con detalle las insoslayables diferencias que existen entre un método, por otro lado tan cuestionado en $\mathrm{ACT}$, como la parada de pensamiento y las estrategias de supresión de pensamiento típicamente usadas en experimentos como los de Wegner. Igualmente, aunque la reevaluación 
usada en los estudios presentados posee elementos característicos o esenciales de la reestructuración cognitiva clásica ésta es, ciertamente, mucho más compleja y amplia.

Por su parte, las técnicas de mindfulness y aceptación deben perfilarse mucho más en protocolos claros, definidos y replicables en varios experimentos, aspecto este también apuntado por Wells (2002). Resulta demasiado genérico mencionar que se siguieron $X$ sesiones de mindfulness, lo que provocó una mejoría de los pensamientos intrusos: es imprescindible una concreción de los contenidos y formatos de estas sesiones, qué aspectos se enfatizaron en ellas y qué efectos concretos tuvieron en los participantes.

Otro tanto cabe decir respecto a la aceptación, pues no es igual un tratamiento que la promueva con metáforas y ejercicios específicamente diseñados, que ofrecer a los participantes únicamente unas indicaciones para aceptar y no modificar los pensamientos que les sobrevengan. Es importante recordar que para la ACT o la BA la aceptación del malestar o de un recuerdo no es un fin en sí misma, sino que está al servicio de los valores de la persona (Hayes et al., 2006), y que se promueve en tanto en cuanto desbloquea la lucha contra los pensamientos o sentimientos en que se halla el paciente para facilitar que retome una vida dirigida a unos objetivos.

Junto con esta pega, debe mencionarse que, en general, los estudios no han diferenciado adecuadamente entre los resultados obtenidos en población clínica y sujetos no clínicos. Sin embargo, Najmi et al. (2010) comprobaron que mientras que los sujetos no clínicos aprendían a través de una demostración ejemplificada la inutilidad de tratar de no pensar en algo, los participantes aquejados de un trastorno obsesivo-compulsivo no cambiaron sus creencias y seguían opinando que era posible controlar la mente de forma directa.

Con todo, la mayor concreción de los métodos de aceptación y mindfulness puede ser un camino que facilite la incorporación de estas técnicas a los tratamientos cognitivo-conductuales estandarizados, como es el caso de la exposición con prevención de respuesta en los cuadros obsesivos, para los que ya se ha sugerido una beneficiosa inclusión del mindfulness que favorece la prevención de respuesta (Fairfax, 2008).

Aunque la lucha por desembarazarse de un pensamiento, una sensación o una emoción determinada han sido metas de la terapia de conducta, no siempre ha sido así en todos los casos, y se ha entendido la utilidad de aceptar estos eventos y seguir activo — piénsese en el ejemplo paradigmático del dolor crónico-. Por tanto, una mejor especificación de los nuevos métodos y su incorporación al bagaje de procedimientos cognitivo-conductuales puede resultar una vía muy prometedora, sin que esto suponga una violación de los principios de los modelos de tercera generación.

\section{Referencias}

Abromowitz, J. S., Tolin, D. F., \& Street, G. P. (2001). Paradoxical effects of thought suppression: a meta-analysis of controlled studies. Clinical Psychology Review, 21, 683703.

Anderson, M. C., \& Green, C. (2001). Suppressing unwanted memories by executive control. Nature, 410, 366-369. 
Bakker, G. M. (2008). Practical CBT: Using functional analysis and standardized homework in everyday therapy. Brisbane: Australian Academic Press.

Bakker, G. M. (2009). In defence of thought stopping. Clinical Psychologist, 13, 59-68.

Barraca, J. (2006). Editorial. Las terapias de conducta de tercera generación: ¿parientes políticos o hermanos carnales? Edupsykhé, 5, 147-157.

Barraca, J. (2009). Habilidades clínicas en la terapia conductual de tercera generación. Clínica y Salud, 20, 109-117.

Beck, A. T. (1976) Cognitive therapy and the emotional disorders. New York: Penguin Books.

Beck, A. T., Emery, G., \& Greenberg, R. L. (1985). Anxiety disorders and phobias: A cognitive perspective. New York: Basic Books.

Beck, A.T., Rush, J., Shaw, B., \& Emery, G. (1983). Terapia cognitiva de la depresión. Bilbao: Desclée De Brouwer. (Trabajo original publicado en 1979).

Buela-Casal, G., Sierra, J. C., \& Vera-Villarroel, P. E. (2001). Evaluación y tratamiento de los Trastornos de Ansiedad II: Fobias simples y fobia social. En G. Buela-Casal, \& J. C. Sierra (Eds.), Manual de evaluación y tratamientos psicológicos (pp. 189-215). Madrid: Biblioteca Nueva.

Cioffi, D., \& Holloway, J. (1993). Delayed costs of suppressed pain. Journal of Personality and Social Psychology, 64, 274-282.

Clark, D. M., Ball, S., \& Pape, D. (1991). An experimental investigation of thought suppression. Behaviour Research and Therapy, 29, 253-257.

Clarck, D. M., \& Purdon, C. (2009). Mental Control of Unwanted Intrusive Thoughts: A Phenomenological Study of Nonclinical Individuals. International Journal of Cognitive Therapy, 2, 267-281.

Dobson, K. S. (1989). A meta-analysis of the efficacy of cognitive therapy for depression.
Journal of Consulting and Clinical Psychology, 57, 414-419.

Dowd, E. T., \& Swoboda, J. S. (1984). Paradoxical interventions in behavior therapy. Journal of Behavior Therapy and Experimental Psychiatry, 15, 229-234.

Dunn, B. D., Billotti, D., Murphy, V., \& Dalgleish, T. (2009). The consequences of effortful emotion regulation when processing distressing material: A comparison of suppression and acceptance. Behaviour Research and Therapy, 47, 761-773.

Ellis, A. (1962). Reason and emotion in psychotherapy. New York: Lyle Stewart.

Ellis, A., \& Bernard, M. E. (Eds.). Clinical applications of rational-emotive therapy. New York: Plenum.

Ellis, A., \& Dryden, W. (1987). The practice of rational-emotive therapy. New York: Springer.

Fairfax, H. (2008). The use of mindfulness in obsessive compulsivedisorder:suggestions for its application and integration in existing treatment. Clinical Psychology \& Psychotherapy, 15, 53-59.

Feldman, G., Greeson, J., \& Senville, J. (2010). Differential effects of mindful breathing, progressive muscle relaxation, and lovingkindness meditation on decentering and negative reactions to repetitive thoughts. Behaviour Research and Therapy, 48, 10021011.

Foa,E.B.,\&Franklin,M.E.(2002).Psychotherapies for obsessive-compulsive disorder: A review. En M. Maj, A. Okasha, N. Sartorius, \& J. Zohar (Eds.), Obsessive-Compulsive Disorder ( $2^{\text {nd }}$ ed.) (pp. 93-115). Chichester, West Sussex: John Wiley \& Sons.

Frankl, V. (1967). Psychotherapy and existentialism: Selected papers on logotherapy. New York: Simon and Shuster.

Gaudiano, B. A. (2009). Öst's (2008) methodological comparison of clinical trials of acceptance and commitment 
therapy versus cognitive behavior yherapy: Matching apples with oranges? Behaviour Research and Therapy, 47, 1066-1070.

Gortner, E. T., Gollan, J. K., Dobson, K. S., \& Jacobson, N. S. (1998). Cognitivebehavioral treatment for depression: Relapse prevention. Journal of Consulting and Clinical Psychology, 66, 377-384.

Hannan, S. E., \& Tolin, D. F. (2005). Acceptance and mindfulness-based behavior therapy for obsessive-compulsive disorder. En S. M. Orsillo, \& L. Roemer (Eds.), Acceptance and mindfulness-based approaches to anxiety: Conceptualization and treatment (pp. 271299). New York: Springer.

Hanstead, M., Gidron, Y., \& Nyklíek, I. (2008). The effects of a mindfulness intervention on obsessive-compulsive symptoms in a nonclinical student population. The Journal of Nervous and Mental Disease, 196, 776-779.

Hayes, S.C.(2004).Acceptanceand commitment therapy, relational frame theory, and third wave of behavioral and cognitive therapies. Behavior Therapy, 35, 639-665.

Hayes, S. C., Jacobson, N. S., Follette, V. M., \& Dougher, M. J. (Eds.). (1994). Acceptance and change: Content and context in psychotherapy. Reno, NV: Context Press.

Hayes, S. C., Luoma, J. B., Bond, F. W., Masuda, A., \& Lillis, J. (2006). Acceptance and Commitment Therapy: Model, process and outcomes. Behaviour Research and Therapy, 44, 1-25.

Hayes, S. C., Strosahl, K. D., \&Wilson, K. G. (1999). Acceptance and Commitment Therapy: An experiential approach to behavior change. New York: Guilford Press.

Hayes, S. C., Wilson, K. W., Gifford, E. V., Follette, V. M., \& Strosahl, K. (1996). Experiential avoidance and behavioral disorders: A functional dimensional approach to diagnosis and treatment. Journal of Consulting and Clinical Psychology, 64, $1152-$ 1168
Hertel, P. T., \& Calcaterra, G. (2005). Intentional forgetting benefits from thought substitution. Psychonomic Bulletin \& Review, 12, 497-509.

Jacobson, N. S., Dobson, K. S. Truax, P. A., Addis, M. E., Koerner, K., Gollan, J. K.,... Prince, S. E. (1996). A component analysis of cognitivebehavioral treatment for depression. Journal of Consulting and Clinical Psychology, 64, 295-304.

Jacobson, N. S., Martell, C. R., \& Dimidjian, S. (2001). Behavioral activation treatment for depression: Returning to contextual roots. Clinical Psychology: Science and Practice, 8, 255-270.

Jacobson, N. S., \& Christensen, A. (1996). Integrative couple therapy: Promoting acceptance and change. New York: Norton.

Kabat-Zinn, J. (1990). Full catastrophe living: Using the wisdom of your body and mind to face stress, pain, and illness. New York: Delta.

Kanter, J. W., Manos, R. C., Bowe, W. M., Baruch, D. E., Busch, A. M., \& Rusch, L. C. (2010). What is behavioral activation? A review of the empirical literature. Clinical Psychology Review, 30, 608-620.

Killingsworth, M. A., \& Gilbert, D. T. (2010). A wandering mind is an unhappy mind. Science, 330, 932.

Kimura, H. (2004). The effects of suppressing unwanted thoughts and replacement strategy. Japanese Journal of Educational Psychology, 52, 115-126.

Klinger, E. (1978). Modes of normal conscious flow. En K. S. Pope, \& J. L. Singer (Eds.), The stream of consciousness (pp. 226-258). New York: Plenum.

Klinger, E. (1996). The contents of thoughts: Interference as the downside of adaptive normal mechanisms in thought flow. En I. G. Sarason, G. R. Pierce, \& B. R. Sarason (Eds.), Cognitive interference: Theories, methods, and findings. (pp. 3-23). Mahwah, NJ: Lawrence Erlbaum Associates. 
Kohlenberg, R. J., \& Tsai, M. (1991). Functional Analytic Psychotherapy: A guide for creating intense and curative therapeutic relationships. New York: Plenum.

Harrington, J. A. (2008). The effectiveness of thought control versus acceptance-based interventions for chronic rumination. Dissertation Abstract International: Section B, 69(6-B), 3846.

Hepburn, S. R., Crane, C., Barnhofer, T., Duggan, D. S., Fennell, M. J. V., \& Williams, J. M. G. (2009). Mindfulness-based cognitive therapy may reduce thought suppression in previously suicidal participants: Findings from a preliminary study. British Journal of Clinical Psychology, 48, 209-215.

Hofmann, S. G., Heering, S., Sawyer, A. T., \& Asnaani, A. (2009) How to handle anxiety: The effects of reappraisal, acceptance, and suppression strategies on anxious arousal. Behavior Research and Therapy, 47, 389394.

Hofmann, S. G., Sawyer, A. T., Witt, A. A., \& Oh, D. (2010). The effect of mindfulness-based therapy on anxiety and depression: A meta-analytic review. Journal of Consulting and Clinical Psychology, 78, 169-183.

Holmes, P., Georgescu, S., \& Liles, W. (2006). Further delineating the applicability of acceptance and change to private responses: The example of dialectical behavior therapy. The Behavior Analyst Today, 7, 311-324.

Howell, A., \& Conway, M. (1992). Mood and the suppression of positive and negative selfreferent thoughts. Cognitive Therapy and Research, 16, 535-555.

Lejuez, C.W., Hopko, D. R., Acierno, R., Daughters, S. B., \& Pagoto, S. L. (2011). Ten year revision of the brief behavioral activation treatment for depression (BATD): Revised treatment manual (BATD-R). Behavior Modification, 35, $111-161$
Lejuez, C. W., Hopko, D. R., \& Hopko, S. D. (2001). A brief behavioral activation treatment for depression: Treatment manual. Behavior Modification, 25, 225-286.

Levin, M., \& Hayes, S. C. (2009). Is acceptance and commitment therapy superior to established treatment comparisons? Psychotherapy \& Psychosomatics, 78, 73-80.

Linehan, M. (1993). Cognitive-behavioral treatment of borderline personality disorder. New York: Guilford Press.

Luciano, J. V., \& Algarabel, S. (2007). Analysis of the efficacy of different thought suppression strategies. International Journal of Psychology and Psychological Therapy, 7, 335-345.

Magee, J. C., \& Teachman, B. A. (2007). Why did the white bear return? Obsessivecompulsive symptoms and attributions for unsuccessful thought suppression. Behaviour Research and Therapy, 45, 28842898.

Marcks, B.A.,\&Woods,D.W.(2005).Acomparison of thought suppression to an acceptancebased technique in the management of personal intrusive thoughts: A controlled evaluation. Behaviour Research and Therapy, 43, 433-445.

Marcks, B. A., \& Woods, D. W. (2007). Role of thought-related beliefs and coping strategies in the escalation of intrusive thoughts: An analog to obsessivecompulsive disorder. Behaviour Research and Therapy, 45, 2640-2651.

Martell, C. R., Addis, M. E. y Jacobson, N. S. (2001). Depression in context: Strategies for guided action. New York: W. W. Norton.

Martell, C. R., Dimidjian, S., \& Herman-Dunn, R. (2010). Behavioral activation for depression: A clinician's guide. New York: Guilford.

Martin, G., \& Pear, J. (2008). Modificación de conducta. Qué es y cómo aplicarla. Madrid: Pearson. 
Masuda, A., Hayes, S. C., Sackett, C. F., \& Twohig, M. P. (2004). Cognitive defusion and selfrelevant negative thoughts: Examining the impact of a ninety year old technique. Behavior Research and Therapy,42, 477-485.

Masuda, A., Hayes, S. C., Twohig, M. P., Drossel, C., Lillis, J., \& Washio, Y. (2009). A parametric study of cognitive defusion and the believability and discomfort of negative self-relevant thoughts. Behavior Modification, 33, 250-262.

Masuda, A., Twohig, M.P., Stormo, A.R., Feinstein, A. B., Chou, Y., \& Wendell, J. W. (2010). The effects of cognitive defusion and thought distraction on emotional discomfort and believability of negative self-referential thoughts. Journal of Behavior Therapy and Experimental Psychiatry, 41, 11-17.

Mazzucchelli, T., Kane, R., \& Rees, C. (2009). Behavioral Activation treatment for adults: A meta-analysis and review. Clinical Psychology: Science and Practice, 16, 383411.

McKay, M., Davis, M., \& Fanning, P. (1985). Técnicas cognitivas para el tratamiento del estrés. Barcelona: Martínez Roca.

Miklowitz, D. J., Alatiq, Y., Goodwin, G. M., Geddes, J. R., Fennell, M. V. F., Dimidjian, S., Hauser, M., \& Williams, J. M. G. (2010). A pilot study of mindfulness-based cognitive therapy for bipolar disorder. International Journal of Cognitive Therapy, 2, 373-382.

Najmi, S., Rees, H., Wilhelm, S., Fama, J., Beck, C., \&Wegner, D. (2010). Learning the Futility of the Thought Suppression Enterprise in Normal Experience and in ObsessiveCompulsive Disorder. Behavioural and Cognitive Psychotherapy, 38, 1-14.

Najmi, S., Riemann, B. C., \&Wegner, D. M. (2009). Managing unwanted intrusive thoughts in obsessive-compulsive disorder: relative effectiveness of suppression, focused distraction, and acceptance. Behaviour Research and Therapy, 47, 494-503.
Najmi, S., \& Wegner, D. M. (2008). The gravity of unwanted thoughts: asymmetric priming effects in thought suppression. Consciousness \& Cognition: An International Journal, 17, 114-124.

Öst, L. G. (2008). Efficacy of the third wave of behavioral therapies: A systematic review and meta-analysis. Behaviour Research and Therapy, 46, 296-321.

Pérez-Álvarez, M. (2006). La terapia de conducta de tercera generación. Edupsykhé, 5, 159172.

Powers, M. B., Zum Vorde Sive Vording, M. B., \& Emmelkamp, M. P. (2009). Acceptance and Commitment Therapy: a meta-analytic review. Psychotherapy and Psychosomatics, 78, 73-80.

Purdon, C. L., \& Clarck, D. A. (2000). White bears and other elusive intrusions: Assessing the relevance of thought suppression for obssesional phenomena. Behavior Modification, 24, 425-253.

Purdon, C. L., Rowa, K., \& Antony, M. M. (2005). Thought suppression and its effects on thought frequency, appraisal and mood state in individuals with obsessivecompulsive disorder. Behaviour Research and Therapy, 43, 93-108.

Quiroga, E., \& Errasti, J. M. (2003). Gruía de tratamientos psicológicos eficaces para los trastornos de la personalidad. En M. Pérez, J. R. Fernández, C. Fernández \& I. Amigo (Coords.), Guía de tratamientos psicológicos eficaces I (pp. 405-428). Madrid: Pirámide.

Rassin, E. (2005). Thought suppression. New York: Elsevier.

Rimm, D. C., \& Masters, J. C. (1979). Behavior therapy: Techniques and empirical findings ( $2^{\text {nd }}$ ed.). New York: Academic Press.

Salkovskis, P. M., \& Campbell, P. (1994). Thought suppression induces intrusion in naturally occurring negative intrusive thoughts. Behaviour Research and Therapy, 32, 1-8. 
Salkovskis, P. M., \& Reynolds, M. (1994). Thought suppression and smoking cessation. Behaviour Research and Therapy, 32, 193201.

Salkovskis, P. M., Thorpe, S. J., Wahle, K., Wroe, A. L., \& Forrester, E. (2003). Neutralizing increases discomfort associated with obsessional thoughts: an experimental study with obsessional patients. Journal of Abnormal Psychology, 112, 709-715.

Segal, Z. V., Williams, J. M. G., \& Teasdale, J. D. (2002). Mindfulness-based cognitive therapy for depression. Nueva York: Guilford Press.

Shingler, J. (2009). Managing intrusive risky thoughts: What Works? Journal of Sexual Aggression, 15, 39-53.

Teasdale, J. D., Segal, Z. V., Williams, J. M. G., Ridgeway, V., Soulsby, J., \& Lau, M. (2000). Prevention of relapse/recurrence in major depression by mindfulness-based cognitive therapy. Journal of Consulting and Clinical Psychology, 68, 615-623.

Vallejo, M. A. (2006). Atención plena. Edupsykhé, 5, 231-253.

Wegner, D. M. (1994). Ironic processes of mental control. Psychological Review, 101, 34-52.

Wegner, D. M., \& Erber, R. (1992). The hyperaccessibility of suppressed thoughts. Journal of Personality and Social Psychology, 63, 903-912.

Wegner, D. M., Schneider, D. J., Carter, S. R., \& White, T. L. (1987). Paradoxical effects of thought suppression. Journal of Personality and Social Psychology, 53, 5-13.

Wegner, D. M., \& Zanakos, S. (1994). Chronic thought suppression. Journal of Personality, 62, 615-640.

Wells, A. (2002). GAD, meta-cognition, and mindfulness: An information processing analysis. Clinical Psychology: Science and Practice, 9, 95-100.

Wenzlaff, R. M., \&Wegner, D. M. (2000). Thought suppression and memory biases during and after depressive moods. Cognition and Emotion, 16, 403-422.

Wiliams, J. M. G., Alatiq, Y., Crane, C. Barnhofer, T., Fennell, M. J.V., Duggan, D. S. et al. (2008). Mindfulness-based cognitive therapy (MBCT) in bipolar disorder. Preliminary evaluation of immediate effects on between-episode functioning. Journal of Affective Disorders, 107, 275-279.

Wilkinson-Tough, M., Bocci, L., Thome, K., \& Herlihy, J. (2010). Is mindfulness-based therapy an effective intervention for obsessive-intrusive thoughts: A case series. Clinical Psychology and Psychotherapy, 17, 250-268.

Wolpe, J. (1958). Psychotherapy by reciprocal inhibition. Standford: Stanford University Press.

Wolpe, J. (1973). The practice of behavior therapy. New York: Pergamon Press.

Wolpe, J. (1992). The practice ofbehavior therapy. Needhan Heights, MA: Allyn \& Bacon. 
